\title{
Sexual Orientation Demographic Data in a Clinical Cohort of Transgender Patients
}

\author{
Samuel Dubin ${ }^{1}$ Tiffany E. Cook ${ }^{1} \quad$ Asa Radix $^{2}$ Richard E. Greene ${ }^{1}$ \\ ${ }^{1}$ NYU Langone Health, New York University School of Medicine, New \\ York, New York, United States \\ ${ }^{2}$ Callen Lorde Community Health Center, NYU Grossman School of \\ Address for correspondence Samuel Dubin, MD, NYU Langone \\ Health, NYU Grossman School of Medicine, 550 1st Avenue, New York \\ City, NY 10010, United States (e-mail: Samuel.dubin@nyumc.org).
} Medicine, New York City, New York, United States

Appl Clin Inform 2021;12:222-228.

\section{Abstract}

Keywords

- sexual orientation

- gender identity

- transgender

- electronic health record
Background There are specific issues regarding sexual orientation (SO) collection and analysis among transgender and nonbinary patients. A limitation to meaningful $\mathrm{SO}$ and gender identity $(\mathrm{GI})$ data collection is their consideration as a fixed trait or demographic data point.

Methods A de-identified patient database from a single electronic health record (EHR) that allows for searching any discrete data point in the EHR was used to query demographic data (sex assigned at birth and current $\mathrm{Gl}$ ) for transgender individuals from January 2011 to March 2020 at a large urban tertiary care academic health center. Results A cohort of transgender individuals were identified by using EHR data from a two-step demographic question. Almost half of male identified $(46.70 \%, n=85)$ and female identified $(47.51 \%, n=86)$ individuals had "heterosexual/straight" input for SO. Overall, male and female identified (i.e., binary) $\mathrm{Gl}$ aggregate categories had similar SO responses. Assigned male at birth (AMAB) nonbinary individuals $(n=6)$ had "homosexual/gay" SO data input. Assigned female at birth (AFAB) nonbinary individuals $(n=56)$ had almost half "something else" SO data input $(41.67 \%, n=15)$. Individuals with "choose not to disclose" for $\mathrm{Gl}(n=249)$ almost all had "choose not to disclose" SO data $(96.27 \%, n=232)$.

Conclusion Current SO categories do not fully capture transgender individuals' identities and experiences, and limit the clinical and epidemiological utility of collecting this data in the current form. Anatomical assumptions based on SO should be seen as a potential shortcoming in over-reliance on $\mathrm{SO}$ as an indicator of screening needs and risk factors.

\section{Background and Significance}

The practice of routine clinical collection of sexual orientation (SO) and gender identity (GI) data to elucidate epidemiological health behavior patterns and inequities among sexual and gender minority (SGM) populations is an emerging and ongoing effort. The field of SOGI data collection has established best practices since the original call for data collection by the National Academy of Medicine and the goal's inclusion in the agendas of healthy people and the National Institutes of Health. ${ }^{1-4}$ For cisgender lesbian, gay, and bisexual (LGB) patients as well as transgender and nonbinary (TGNB) patients, the collection of SOGI data are a means to increase access by affirming these identities in clinical spaces, improve research and quality improvement efforts to support these populations, and remind providers received

August 24, 2020

accepted after revision

January 19, 2021 (c) 2021. Thieme. All rights reserved. Georg Thieme Verlag KG,

Rüdigerstraße 14,

70469 Stuttgart, Germany
DOI https://doi.org/

10.1055/s-0041-1725184. ISSN 1869-0327. 
that different risk behaviors, health screenings, and psychosocial stressors should be addressed in SGM individuals.

There is consensus on the importance of SOGI data collection. ${ }^{2,5}$ However, within this same body of research, there are documented challenges to current methods of categorization. Scheffey et al documented the patient preference for "more flexible (SO and $\mathrm{GI}$ ) options...(that) provide a more accurate reflection of individual identities," noting how this can hinder SOGI utility for epidemiological study. ${ }^{6}$ Another study of provider attitudes documented provider prioritization of sexual behavior or SO, citing behavior's more immediate relevance to clinical care in the context of limited encounter time. ${ }^{7}$ The importance of SOGI collection specifically for TGNB patients has also been documented, including the challenges of a standardized vocabulary and population definitions. ${ }^{5,8}$

One criticism of SO clinical categories is their inclusion of multiple constructs-attraction, behavior, and identitywithin a single label. ${ }^{9}$ Research documenting the potential for patients to only report behavior consistent with stated identity concluded that SO data collection does not replace the need to query sexual behavior. ${ }^{7}$ Similar findings have been demonstrated in a United States survey of 5,000 youth where an appreciable discordance between sexual identity and romantic attraction was noted. ${ }^{10}$ One study of university students' perspectives on SOGI labels in health care settings found that roughly one-third would identify SO differently by context (i.e., health care vs. elsewhere) and roughly onefifth indicated the same for GI. ${ }^{6}$ Dichter and Ogden state the issue succinctly: the labels used for SOGI in a clinical context can signify political affiliation, behavior, or internal identity. ${ }^{11}$

Another potential limitation to meaningful SOGI data collection is that SO status is captured as a fixed trait or demographic data point despite well-documented sexual fluidity. Fluidity of SO, gender expression, and GI, especially within pediatric populations, is considered as part of anticipated developmental trajectories and presents challenges for documentation. ${ }^{12}$ In a prospective sample of over 1,000 adolescents, $41.2 \%$ of the sample endorsed sexual minority status at one point from age 14 to 22 years. ${ }^{13}$ A study of 452 gender minority adults in Massachusetts found that $58.2 \%$ reported experiencing changes in sexual attractions in their lifetime, and $64.6 \%$ among those who had a social gender transition. ${ }^{14}$ An online study of approximately 300 transgender individuals found a mean of more than two current gender identities and approximately two past SOs. ${ }^{15}$ An earlier study of "self-identified female-to-male" individuals documented that $40 \%$ who had begun to transition reported a shift in SO. ${ }^{16}$

In addition to the documented fluidity of GI labels within TGNB communities, there are specific SO collection issues among TGNB patients. ${ }^{17}$ Notably, identifying gender minorities' $\mathrm{SO}$ in the standardized way (i.e., binary, anatomy based, and defaulting to cisgender) creates unique barriers to meaningful SO data as compared with cisgender populations. ${ }^{5,8,18-20}$ Definitions of SO categories depend on an assumed alignment between genitalia and gender for both an individual and their partner. ${ }^{11,14}$ Whether through noncisgender gender identities or anatomical changes for themselves or their partners, current categories fail to meaningfully capture many TGNB individuals' SO.

The need to collect and utilize SO and GI data in the service of TGNB individuals and populations is not rendered moot by the challenges. SO response patterns have been found to significantly vary between "gender congruent and noncongruent gender identities," with significant differences found when disaggregated based on sex assigned at birth. ${ }^{9}$ One study found more symptoms of anxiety among transgender men attracted to both men and women compared with men only. ${ }^{16} \mathrm{~A}$ recent study summarizes: "heterogeneity of sexual orientation identities and sexual fluidity in attractions are the norm rather than the exception among gender minority people." ${ }^{14}$

SO data limitations for TGNB patients curtail the data's clinical and epidemiological utility, which compounds the stark health disparities experienced by these populations. Notably, in the study which helped to validate current SO and GI data response options the conclusions about SO questions accurately reflecting identities were for LGB patients only. ${ }^{2}$ Another limitation is that behavioral risk screening relevant to SGM individuals is not captured by SOGI data alone, and may require more information like anatomical inventory and prior SO and GI experiences. Here, we analyze the responses of SO among a sample of patients identified as TGNB to better characterize responses in clinical settings. Our aim is to identify shortcomings to collect meaningful so data for gender minorities.

\section{Methods}

We performed a de-identified EHR chart review study of patients with health care encounters at NYU Langone Health, an urban academic institution. A database with all discrete data points from the EHR was searched by using Boolean logic and time parameters. ${ }^{21}$ The database also allows searching for several distinct patients within a set of search terms, as well as demographic data including age, race, and language spoken. Patient information in the database is deidentified with a proprietary algorithm by a third-party provider, i2b2. ${ }^{21}$ Patient information is further de-identified by randomly offsetting dates associated with patient data 1 to 90 days forward.

Our institution's EHR is Epic, and an institutionally customized SOGI data collection upgrade was instituted in December 2017. The GI demographic categories are "sex assigned at birth" ( $\mathrm{SAB}$ ) and "current gender identity." The SAB prompt is "what was your sex assigned at birth" and one of the following answer options can be chosen: "male," "female," and "choose not to disclose." Notably, legal sex data input is a separate data point used for billing purposes, is filled based on patient legal documentation, and does not populate the SAB fields. The GI prompt reads "what is your gender identity" and one of the following options can be chosen: male, female, transgender female, transgender male, non-binary, other, and choose not to disclose. Selecting the 
response "other" prompts a free response. The SO prompt reads "what is your current sexual orientation" and one of the following options can be chosen: bisexual, choose not to disclose, don't know, heterosexual/straight, homosexual/ gay, lesbian, and something else. The entered text was not visible to our query using the database searched.

Our institution requires all providers to take a one-time online SOGI data collection-specific training module, even if they are not responsible for collecting the data-with specific submodules based on clinic role (front desk/registration, emergency department, inpatient and procedure area, and other clinical roles). Providers are not mandated to ask SOGI questions at our institution, and information about sexual behavior is collected when providers deem the information relevant to the patient's care.

GI demographic data are input into the EHR during or after a clinical encounter by physicians, nurse practitioners, physician assistants, nurses, and mental health care providers. Patients over 13 years old who have a clinical encounter with gender-affirming surgeons may enter their own GI demographic data as well through a survey in the patient portal (MyChart) at any time once they have patient portal access. The most recent input overwrites prior input, and no record of changes is retained by the system if data are overwritten.

Institutional review board approval to access data was obtained from NYU Langone Health and maintained throughout study period.

\section{Analysis}

All data extraction and analyses were completed in March 2020. The EHR data queried was from January 2011 to March 2020. The search database tallies the number of unique patients that meet search criteria; thus, the results of searches are automatically counted. The database's count results are accurate to plus or minus three unique patients. Percentages were calculated manually.

A combination of SAB of "male" and GI of "male" as well as $S A B$ of "female" and GI of "female" were considered cisgender, that is, alignment between SAB and GI. A SAB of "male" with any GI input other than "male," and a SAB of "female" with any GI input other than "female" was considered as transgender. The limitations of this are discussed below.

For purposes of analysis, GI was grouped according to male, female, or nonbinary identification. Male identified were considered those with a GI of "transmale" or "male" regardless of SAB. Female identified were considered those with a GI of "transfemale" or "female" regardless of SAB. Nonbinary individuals were considered those with a GI input of nonbinary or other, regardless of SAB.

\section{Results}

\section{Demographic Characteristics of the Two-Step Question-Identified Transgender Cohort}

A total of 821 individuals were identified by using EHR data from a two-step GI question. The largest age group of individuals was 18 to 34 years old $(55.73 \%, n=316)$, followed
Table 1 Demographic characteristics of the two-step questionidentified transgender cohort

\begin{tabular}{|c|c|c|}
\hline & $\begin{array}{l}n=\text { Unique } \\
\text { patients }\end{array}$ & $\begin{array}{l}\text { Percentage } \\
\text { (\%) of transgender } \\
\text { individuals }\end{array}$ \\
\hline \multicolumn{3}{|l|}{ Age } \\
\hline $0-9$ & 0 & 0.00 \\
\hline $10-17$ & 64 & 11.29 \\
\hline $18-34$ & 316 & 55.73 \\
\hline $35-44$ & 78 & 13.76 \\
\hline $45-54$ & 48 & 8.47 \\
\hline $55-64$ & 38 & 6.70 \\
\hline $65-74$ & 12 & 2.12 \\
\hline $75-84$ & 7 & 1.23 \\
\hline $85-90$ & 0 & 0.00 \\
\hline \multicolumn{3}{|l|}{ Race } \\
\hline $\begin{array}{l}\text { African American } \\
\text { (Black) }\end{array}$ & 88 & 15.52 \\
\hline Asian & 5 & 0.88 \\
\hline Asian Indian & 5 & 0.88 \\
\hline Chinese & 8 & 1.41 \\
\hline $\begin{array}{l}\text { Native American } \\
\text { (American Indian/ } \\
\text { Eskimo/Aleutian) }\end{array}$ & 9 & 1.59 \\
\hline Other Race & 93 & 16.40 \\
\hline Patient Refused & 4 & 0.71 \\
\hline Unknown & 29 & 5.11 \\
\hline White & 330 & 58.20 \\
\hline
\end{tabular}

by 35 to 44 years $(13.76 \%, n=78$; - Table 1$)$. For age and race breakdown, see - Table 1 .

Of this cohort, $37.76 \%(n=310)$ were assigned male at birth (AMAB) and $62.24 \%(n=511)$ were assigned female at birth (AFAB). Between a quarter and one-third of individuals (26.77\% of $A M A B$ and $31.90 \%$ of $A F A B$ individuals) had "choose not to disclose" as their GI input (-Figs. 1 and 2). Male identified individuals comprised $35.83 \%(n=292)$ of overall respondents, and female identified individuals comprised $26.01 \%(n=212)$ of overall respondents (-Table 2 ). Respondents with a GI of "choose not to disclose" comprised $30.55 \%$ ( $n=249)$ of the overall sample. The remaining nonbinary (GI of "non-binary" or "other") were predominantly $\operatorname{AFAB}(n=56$, vs. $n=6$ AMAB). (-Table 2 )

There were 13 AFAB individuals (2.54\% of all AFAB individuals) who identified as transfemales and 19 AMAB individuals $(6.13 \%$ of all AMAB individuals) who identified as transmales (While these are valid and possible, they may represent limitations of data collection which we discuss below). For the breakdown of GI responses by sex assigned at birth, please see - Figs. 1 and 2. 


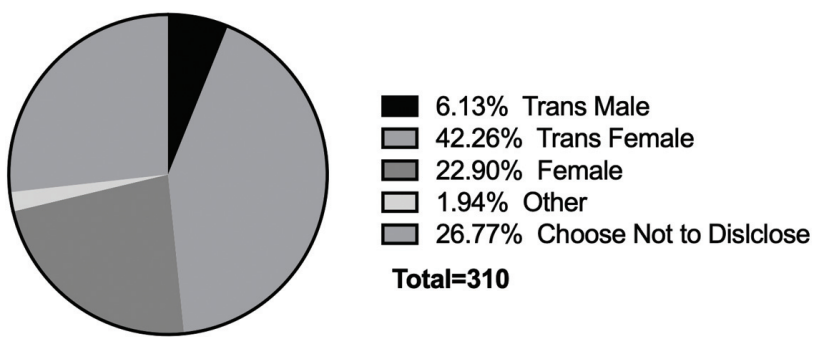

Fig. 1 Gender identity responses for sex assigned male at birth individuals. Note: Nonbinary is not displayed because it had a value of zero percent.

\section{The Amount of Missing and Present Sexual Orientation Data Vary Among Gender Identity Groupings}

The overall presence of SO data for binary, nonbinary, and individuals with current GI "choose not to disclose" input was $77.22 \%$. The GI categories with the most missing SO data were "male identified" $(n=292,62.33 \%)$ and "nonbinary and other: AFAB" ( $n=56,64.29 \%)$ (-Table 2$)$. The completed responses "nonbinary and other: $A M A B$ " $(n=6)$ were $100 \%$ and for "choose not to disclose (AMAB and AFAB)" were 96.79\% ( $n=249$; -Table 2$)$.

\section{Sexual Orientation Responses of Transgender Individuals}

Almost half of male identified (transmale or male GI; $46.70 \%$, $n=85$ ) and female identified (transfemale or female GI; $47.51 \%, n=86$ ) individuals had "heterosexual/straight" input for SO (-Table 3). Overall, these two binary GI aggregate categories (male and female identified) had similar SO responses; following "heterosexual/straight" was "bisexual," then "don't know," then "homosexual/gay," then "something else," and then "choose not to disclose" (- Table 3). Notably, no binary individuals had "lesbian" input for SO ( - Table 3 ).

Individuals with a nonbinary GI, here the GI options "nonbinary" and "other," had variation by sex assigned at birth. AMAB nonbinary individuals (GI of nonbinary and other; $n=6$ ) all had "homosexual/gay" SO data input ( - Table 3). AFAB nonbinary individuals (GI of nonbinary and other; $n=56$ ) had almost half "something else" SO data input (41.67\%, $n=15$; - Table 3 ).
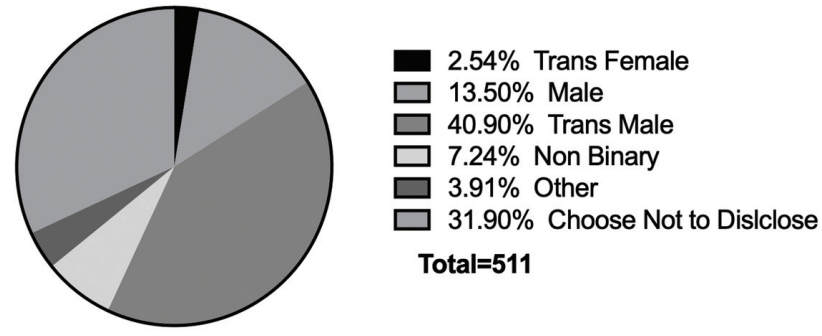

Fig. 2 Gender identity responses for sex assigned female at birth individuals.

Individuals with "choose not to disclose" for GI $(n=249)$ almost all had "choose not to disclose" SO data (96.27\%, $n=232$; -Table 3 ). The only other SO category with a response for "choose not to disclose" GI was a SO of "heterosexual/straight" $(n=9,3.73 \%)$. We discuss possible reasons for this below.

\section{Discussion}

As part of an ongoing SO and GI data collection implementation at our institution, we identified a cohort of transgender individuals using a two-step GI demographic question to examine SO responses. In the study widely cited as the validation of current SOGI data response options, the conclusions about SO questions accurately reflecting identities were for LGB patients only. ${ }^{2}$ To our knowledge, there are no studies that have identified an entirely clinical population of transgender individuals to analyze SO responses. Our analysis seeks to assess the clinical utility of these SO responses among gender minorities. The shortcomings of $\mathrm{SO}$ responses for transgender individuals undermine these data's usefulness in clinical settings.

For specific disaggregated transgender binary and nonbinary populations, we found high rates of responses for SO options “Something else” and “don't know." For AFAB nonbinary individuals, the most common response was "something else" (41.67\%), followed by "lesbian" (41.67\%), with "don't know" (13.89\%) and "homosexual/gay" (13.89\%) tied for the third most common response (- Table 3). In our EHR, selecting "something else" for SO prompts an optional free

Table 2 Gender identity aggregate categories

\begin{tabular}{|l|l|l|l|}
\hline Gender identity aggregate category & $\begin{array}{l}n=\text { Total individuals within } \\
\text { gender identity } \\
\text { aggregate category }\end{array}$ & $\begin{array}{l}\text { Gender identity } \\
\text { category \% of } \\
\text { total individuals }\end{array}$ & $\begin{array}{l}\text { \% with input } \\
\text { (i.e., not missing) } \\
\text { SO data }\end{array}$ \\
\hline Male identified $^{\text {a }}$ & 292 & 35.83 & 62.33 \\
\hline Female identified $^{\text {b }}$ & 212 & 26.01 & 85.38 \\
\hline Nonbinary and other: AFAB & 56 & 6.87 & 64.29 \\
\hline Nonbinary and other: AMAB & 6 & 0.74 & 100.00 \\
\hline Choose not to disclose: AMAB and AFAB & 249 & 30.55 & 96.79 \\
\hline
\end{tabular}

Abbreviations: AFAB, assigned female at birth; $A M A B$, assigned male at birth.

${ }^{\mathrm{a}}$ Transmale (either sex assigned at birth), male (assigned female at birth).

${ }^{\mathrm{b}}$ Transfemale (either sex assigned at birth), female (assigned male at birth). 
Table 3 Sexual orientation responses of transgender and nonbinary individuals

\begin{tabular}{|c|c|c|c|}
\hline Gender identity & Sexual orientation & $n=$ individuals & $\begin{array}{l}\text { Percentage }{ }^{a} \text { of gender } \\
\text { identity category (\%) }\end{array}$ \\
\hline \multirow{7}{*}{$\begin{array}{l}\text { Male identified } \\
\text { (transmale and } \\
\text { male) }\end{array}$} & Bisexual & 27 & 14.84 \\
\hline & Choose not to disclose & 9 & 4.95 \\
\hline & Don't know & 25 & 13.74 \\
\hline & Heterosexual/straight & 85 & 46.70 \\
\hline & Homosexual/gay & 20 & 10.99 \\
\hline & Lesbian & 0 & 0.00 \\
\hline & Something else & 16 & 8.79 \\
\hline \multirow{7}{*}{$\begin{array}{l}\text { Female identified } \\
\text { (transfemale and } \\
\text { female) }\end{array}$} & Bisexual & 25 & 13.81 \\
\hline & Choose not to disclose & 8 & 4.42 \\
\hline & Don't know & 24 & 13.26 \\
\hline & Heterosexual/straight & 86 & 47.51 \\
\hline & Homosexual/gay & 23 & 12.71 \\
\hline & Lesbian & 0 & 0.00 \\
\hline & Something else & 15 & 8.29 \\
\hline \multirow{7}{*}{$\begin{array}{l}\text { Nonbinary and oth- } \\
\text { er: assigned female } \\
\text { at birth }\end{array}$} & Bisexual & 4 & 11.11 \\
\hline & Choose not to disclose & 0 & 0.00 \\
\hline & Don't know & 5 & 13.89 \\
\hline & Heterosexual/straight & 0 & 0.00 \\
\hline & Homosexual/gay & 5 & 13.89 \\
\hline & Lesbian & 7 & 19.44 \\
\hline & Something else & 15 & 41.67 \\
\hline \multirow{7}{*}{$\begin{array}{l}\text { Nonbinary and oth- } \\
\text { er: assigned male at } \\
\text { birth }\end{array}$} & Bisexual & 0 & 0.00 \\
\hline & Choose not to disclose & 0 & 0.00 \\
\hline & Don't know & 0 & 0.00 \\
\hline & Heterosexual/straight & 0 & 0.00 \\
\hline & Homosexual/gay & 6 & 100.00 \\
\hline & Lesbian & 0 & 0.00 \\
\hline & Something else & 0 & 0.00 \\
\hline \multirow{7}{*}{$\begin{array}{l}\text { Choose not to } \\
\text { disclose }\end{array}$} & Bisexual & 0 & 0.00 \\
\hline & Choose not to disclose & 232 & 96.27 \\
\hline & Don't know & 0 & 0.00 \\
\hline & Heterosexual/straight & 9 & 3.73 \\
\hline & Homosexual/gay & 0 & 0.00 \\
\hline & Lesbian & 0 & 0.00 \\
\hline & Something else & 0 & 0.00 \\
\hline
\end{tabular}

aPercentages are calculated among respondents with the same gender identity.

text response. Given the lack of validated so responses among transgender individuals, it is possible "don't know" respondents did not see their preferred SO identity as an option, truly do not know, or it could reflect the larger failure of SO categories to meaningfully apply to some transgender individuals' experiences. Additionally, there are many valid clinical scenarios, such as in urgent or emergent settings, where patients are unable to answer demographic data information at the time of intake into health care system.

Individuals with a GI of "choose not to disclose" almost exclusively had "choose not to disclose" as their SO data input ( $\sim$ Table 2). Given that we limited our analysis to individuals identified by demographic data only (vs. including transgender-specific diagnostic codes), we assume that whomever is 
entering the data are either not asking the patient or that the patient (who may also be entering their data through an online portal) is unwilling to disclose this demographic information. Staff reluctance to ask patients SO and GI questions may arise when a patient's gender presentation does not align with the staff's assumptions of their "correct" demographic data.

The high concordance between GI and SO responses of "choose not to disclose" could also be a phenomenon in response to insurance billing practices, which frequently require a binary GI to authorize gender affirming surgery. If that is the case, the non-binary cohort identified is likely an underestimate. Alternatively, this may reflect patients' reluctance to document this data due to security or disclosure concerns. Regardless of the reason why, the fact that 30.55\% of our cohort had a GI of "Choose Not to Disclose" reflects the need to improve on data collection efforts.

SO identity and behaviors are not always congruent. Our results demonstrate that solely relying on SO is insufficient to guide clinical risk and screening assessments. Among binary transgender individuals, "heterosexual/straight" was the most common SO option input $446.70 \%$ of male identified and $47.51 \%$ of female identified individuals, -Table 3). If a clinician assumes behavioral risk factors based on current SO alone, a variety of relevant behavioral interventions or preventative screenings will go unaddressed. Anatomical inventories have been suggested by the World Professional Association of Transgender Health (WPATH)'s EHR working group as a best practice. ${ }^{18}$ Our findings reiterate the importance of an anatomical inventory rather than creating a faulty logical link of assumptions among genitalia, gender, SO, and clinical needs.

Additionally, $\mathrm{SO}$ is a label that includes sexual attraction, sexual behavior, and political identity collapsed into one. For transgender individuals, of whom many in our health system have had contact with gender affirming surgeons, the SO subcategory of sexual behavior assumes an alignment between genitalia, sex, and gender and is a likely point of theoretical slippage. Because of the reliance of SO categories on assumed genitalia of both an individual and their sexual partner(s) (e.g., "same-sex couples" as defined by sex via genitalia), the implied definition of SO for some transgender individuals may be rendered meaningless by gender-affirming anatomical changes. Additionally, for individuals who choose to use hormones or undergo gender affirming surgery, sexual attraction-not just anatomy-may also change. This potential failure of standard SO categories extends to all individuals who are sexually partnering with transgender and/or nonbinary individuals.

Our work has several limitations. Quantifying patients identified as transgender should ideally use dynamic GI data that is collected and reassessed on a routine basis. Our analysis seeks to assess SO responses among transgender individuals in a clinical context. Notably, not all patients identified as transgender had completed SO responses (-Table 2). Overwritten data and clerical errors limit the applicability of our conclusions to response categories with the smallest sample sizes. The absence of overwritten data limits our understanding of how changes in one demographic domain such as GI may impact changes in another, such as SO. While this association is noncausal, the absence of overwritten data limits our understanding of the interplay between GI and SO categories. The incomplete nature of SO responses among transgender individuals further limits our sample size and our conclusion may only be applicable to a subset of transgender individuals within our health care system. Additionally, our inability to query free text responses prevents us from identifying gaps in potential response options. The conclusions drawn here are applicable in scope to a quaternary care, urban hospital system with a gender-affirming surgery and fertility services, but may not be representative of other health systems. Nevertheless, the sample does remain valuable in identifying gaps and limitations in SO and GI data collection questions.

Future research is warranted to identify the most effective ways of using SO data with additional supporting data about anatomy and sexual behaviors to most effectively identify and care for patients. The utility of data from an EHR with the capacity to collect SO and GI data that changes for an individual over time should also be studied further. Future research should also look at SOGI data collection and its concordance with risk behaviors, health screenings, and psychosocial stressors to see if additional formalized screening data can be helpful.

\section{Conclusion}

Collecting SO data on TGNB people highlights that SO data alone do not accurately reflect the experience of this community, especially at one time point. Assessments of risk factors should avoid assumptions about anatomy and gender inherent in SO categories. Anatomical assumptions based on $\mathrm{SO}$ should be seen as a potential shortcoming in over-reliance on SO as an indicator of screening needs and risk factors. SO data collection in all people may fall short of its goal because it does not take into account the changing bodies, identities, and behaviors of all people.

\section{Clinical Relevance Statement}

Current SO categories do not fully capture transgender individuals' identities and experiences, and may limit the utility of collecting this data in the current form. SO options should be documented on a repeated basis. Assessments of risk factors should avoid assumptions about anatomy and gender inherent in SO categories.

\section{Multiple Choice Questions}

1. Which sexual and gender minority populations have validated questions for the collection of SO data?

a. Only gay and lesbian identified individuals.

b. There are no populations that have validated SO questions.

c. Only transgender individuals, regardless of SO.

d. Only cisgender individuals, regardless of SO. 
Correct Answer: D is correct. The main source of validated SO questions, the "do ask, do tell" (Cahill et al 2014) study did not have enough transgender-identifying respondents to make conclusions about SO response options for these populations.

2. Which of the following are encompassed by the term SO?
a. Attraction and behavior
b. Attraction only
c. Attraction, behavior, and identity
d. Identity only
e. Behavior only

Correct Answer: C is correct. SO is a composite of sexual attraction, sexual behavior, and sexual and/or political identity.

\section{Protection of Human and Animal Subjects}

No human subjects were involved in the project.

Funding

None.

\section{Conflict of Interest}

None declared.

\section{Acknowledgments}

The authors would like to thank Aron Janssen, Alison Liss, Glenn Doty, Kevin Moore, and Joey Nicholson.

\section{References}

1 National Academy of Medicine. The Health of Lesbian, Gay, Bisexual, and Transgender (LGBT) People: Building A Foundation for Better Understanding. Washington (D.C.): National Academies Press; 2011

2 Cahill S, Singal R, Grasso C, et al. Do ask, do tell: high levels of acceptability by patients of routine collection of sexual orientation and gender identity data in four diverse American community health centers. PLoS One 2014;9(09):e107104

3 Office of Disease Prevention and Health Promotion. Lesbian, Gay, Bisexual, and Transgender Health |Healthy People. 2020. Accessed December 15, 2019 at: https://www.healthypeople.gov/2020/ topics-objectives/topic/lesbian-gay-bisexual-and-transgenderhealth

4 Pérez-Stable EJ. Sexual and gender minorities formally designated as a health disparity population for research purposes. Accessed December 15, 2019 at: https://www.nimhd.nih.gov/about/directors-corner/messages/message_10-06-16.html

5 Deutsch MB, Buchholz D. Electronic health records and transgender patients-practical recommendations for the collection of gender identity data. J Gen Intern Med 2015;30(06):843847
6 Scheffey KL, Ogden SN, Dichter ME. "The idea of categorizing makes me feel uncomfortable": university student perspectives on sexual orientation and gender identity labeling in the healthcare setting. Arch Sex Behav 2019;48(05):1555-1562

7 Dichter ME, Ogden SN, Scheffey KL. Provider perspectives on the application of patient sexual orientation and gender identity in clinical care: a qualitative study. J Gen Intern Med 2018;33(08): 1359-1365

8 Reisner SL, Deutsch MB, Bhasin S, et al. Advancing methods for US transgender health research. Curr Opin Endocrinol Diabetes Obes 2016;23(02):198-207

9 Bosse JD, Chiodo L. It is complicated: gender and sexual orientation identity in LGBTQ youth. J Clin Nurs 2016;25(2324):3665-3675

10 Ybarra ML, Price-Feeney M, Mitchell KJ. A cross-sectional study examining the (in)congruency of sexual identity, sexual behavior, and romantic attraction among adolescents in the US. J Pediatr 2019;214:201-208

11 Dichter ME, Ogden SN. The challenges presented around collection of patient sexual orientation and gender identity information for reduction of health disparities. Med Care 2019;57(12): 945-948

12 Tan-McGrory A, Bennett-AbuAyyash C, Gee S, et al. A patient and family data domain collection framework for identifying disparities in pediatrics: results from the pediatric health equity collaborative. BMC Pediatr 2018;18(01):18

13 Berona J, Stepp SD, Hipwell AE, Keenan KE. Trajectories of sexual orientation from adolescence to young adulthood: results from a community-based urban sample of girls. J Adolesc Health 2018; 63(01):57-61

14 Katz-Wise SL, Reisner SL, Hughto JW, Keo-Meier CL. Differences in sexual orientation diversity and sexual fluidity in attractions among gender minority adults in Massachusetts. J Sex Res 2016;53(01):74-84

15 Kuper LE, Nussbaum R, Mustanski B. Exploring the diversity of gender and sexual orientation identities in an online sample of transgender individuals. J Sex Res 2012;49(2-3):244-254

16 Meier SC, Pardo ST, Labuski C, Babcock J. Measures of clinical health among female-to-male transgender persons as a function of sexual orientation. Arch Sex Behav 2013;42(03):463-474

17 Coleman E. Assessment of sexual orientation. J Homosex 1987;14 $(1-2): 9-24$

18 Deutsch MB, Green J, Keatley J, Mayer G, Hastings J, Hall AMWorld Professional Association for Transgender Health EMR Working Group. Electronic medical records and the transgender patient: recommendations from the World Professional Association for Transgender Health EMR Working Group. J Am Med Inform Assoc 2013;20(04):700-703

19 Deutsch MB, Keatley J, Sevelius J, Shade SB. Collection of gender identity data using electronic medical records: survey of current end-user practices. J Assoc Nurses AIDS Care 2014;25(06): 657-663

20 Collin L, Reisner SL, Tangpricha V, Goodman M. Prevalence of transgender depends on the "case" definition: a systematic review. J Sex Med 2016;13(04):613-626

21 Murphy SN, Mendis ME, Berkowitz DA, Kohane I, Chueh HC. Integration of clinical and genetic data in the i2b2 architecture. AMIA Annu Symp Proc 2006;2006:1040 